\title{
Breathlessness and crises in the context of advanced illness: A comparison between COPD and lung cancer patients
}

\author{
CHRISTINE DUNGER, M.SC., B.SC.N., ${ }^{1}$ IRENE J. HIGGINSON, B.MED.SCI., PH.D., ${ }^{2}$ \\ MARJOLEIN GYSELS, M.A., PH.D., ${ }^{3}$ SARA BOOTH, M.D., ${ }^{4}$ \\ STEFFEN T. SIMON, M.SC., M.D., ${ }^{2,5,6}$ AND CLAUDIA BAUSEWEIN, M.D., PH.D. ${ }^{2,7}$ \\ ${ }^{1}$ Institute of Ethics and Communication in Health Care, University of Witten/Herdecke, 58453 Witten, Germany \\ ${ }^{2}$ King's College London, Department of Palliative Care, Policy and Rehabilitation, Cicely Saunders Institute, London \\ SE5 9PJ, United Kingdom \\ ${ }^{3}$ Amsterdam Institute of Social Science Research, Amsterdam, The Netherlands \\ ${ }^{4}$ Palliative Care Team, Addenbrooke's Hospital, Cambridge CB2 0QQ, United Kingdom \\ ${ }^{5}$ Department of Palliative Medicine and Clinical Trials Center (BMBF 01KN0706), University Hospital Cologne, 50924 \\ Cologne, Germany \\ ${ }^{6}$ Institute of Palliative Care, 26121 Oldenburg, Germany \\ ${ }^{7}$ Department of Palliative Medicine, University of Munich, 81377 Munich, Germany \\ (RECEIVEd September 12, 2013; AcCEPTEd October 31, 2013)
}

\begin{abstract}
Objective: The objective of this study was to explore and contrast the experience and meaning of breathlessness in patients with chronic obstructive pulmonary disease (COPD) or lung cancer at the end of life.

Method: We conducted a qualitative study embedded in a longitudinal study using topicguided in-depth interviews with a purposive sample of patients suffering from breathlessness affecting their daily activities due to advanced (primary or secondary) lung cancer or COPD stage III/IV. All interviews were audiotaped, transcribed verbatim, and analyzed using framework analysis.

Results: Ten COPD and eight lung cancer patients were interviewed. Both groups reported similarities in their experience. These included exertion through breathlessness throughout the illness course, losses in their daily activities, and the experience of breathlessness leading to crises. The main difference was the way in which patients adapted to their particular illness experience and the resulting crises over time. While COPD patients more likely sought to get their life with breathlessness under control, speaking of daily living with breathlessness under certain conditions, the participating lung cancer patients often faced the possibility of death and expressed a need for security.

Significance of Results: Breathlessness leads to crises in patients with advanced disease. Although experiences of patients are similar, reactions and coping mechanisms vary and are more related to the disease and the stage of disease.
\end{abstract}

KEYWORDS: Dyspnea, Symptom experience, COPD, Cancer, Palliative care, Qualitative

\section{INTRODUCTION}

Breathlessness toward the end of life is experienced by patients and carers as distressing and frightening,

Address correspondence and reprint requests to: Claudia Bausewein, Department of Palliative Medicine, Munich University Hospital, Marchioninistr. 15, 81377 Munich. E-mail: Claudia.bausewein@med.uni-muenchen.de. but at present the ability to manage this condition is still far from optimal (Booth et al., 2009). Breathlessness is defined as "a subjective experience of breathing discomfort that is comprised of qualitatively distinct sensations that vary in intensity" (American Thoracic Society, 1999). The prevalence of breathlessness in cancer patients varies between 15 and $70 \%$ during the final year of life (Solano 
et al., 2006). In chronic obstructive pulmonary disease (COPD), breathlessness is the most dominant symptom. In the US SUPPORT study, around 70\% of patients with COPD experienced moderate to severe breathlessness during the last six months of life, with an increase toward death (i.e., the last three days) to more than $80 \%$ (Lynn et al., 2000). The symptom burden and palliative care needs of breathless patients with severe COPD are considerable and as high as among patients with advanced primary and secondary lung cancer, though patients with COPD have a longer survival (Bausewein et al., 2010b).

Several studies have explored the experience of patients living with breathlessness caused by various conditions. Dyspnea was found to create barriers throughout all aspects of living with terminal cancer, mainly isolating the patient (Roberts et al., 1993). Patients with cancer and COPD and their carers feel restricted in their daily activities and reported that they were very disabled and became increasingly dependent because of breathlessness (Booth et al., 2003). Manifestation of breathlessness was found to be different in cancer and COPD, which was related to differences in disease progression (Booth et al., 2003). The invisibility of breathlessness as experienced by COPD patients was a barrier to access to services for patients, and contact with healthcare was postponed to crisis point (Gysels \& Higginson, 2008; 2010). Acute episodes of breathlessness often led to considerable distress of patients, resulting in crises (Heinzer et al., 2003). For COPD patients, acute exacerbations have been identified as the main reason for acute crises (Ramsenthaler et al., 2012).

Palliative care services for COPD patients are often modeled on the experience professionals have had with cancer patients. This is supported by the view that there is a common pathway toward the end of life in terms of symptomatology, including breathlessness, between different disease groups (Solano et al., 2006). However, little is known about the experience of patients suffering breathlessness in the context of different diseases. Therefore, the aim of the present study was to explore and contrast the experience and the meaning of breathlessness for patients in the context of advanced cancer and severe COPD.

\section{METHODS}

\section{Design}

Qualitative in-depth interviews were conducted with patients suffering from breathlessness due to advanced disease. The interviews were conducted as part of a wider longitudinal study describing the tra-
Table 1. Themes of topic guide

Course of the illness

Experience of living with breathlessness through the illness course

Living with breathlessness: Impact on daily activities Influence on family living, social life, support and the patients themselves

Treatment experiences: Therapy and experience with professionals

Future expectations: Place of care, expectations and awareness of prognosis

jectories of breathlessness (Bausewein et al., 2010a). A topic guide was developed for this part of the study to ensure that relevant topics around the experience of breathlessness and management strategies were covered (see Table 1).

\section{Participants and Setting}

Participants were included in the longitudinal study if suffering from breathlessness that affected their daily activities and suffered from advanced malignant disease (either primary or secondary lung cancer) or stage III/IV COPD. As patients with primary lung cancer often suffer from COPD, these patients were only included if their breathlessness could be clearly related to their cancer diagnoses. A purposive sample of patients participating in the longitudinal study was asked to take part in the interviews. To ensure a sample with maximum variation representing a wide spectrum of experience, interview participants were selected with regard to severity of breathlessness, diagnosis, duration of illness, age, sex, living alone or with caregiver, and place of care (inpatient and community). Patients who lacked the capacity to give informed consent, who were too ill to be interviewed, or who could not speak or read German fluently were excluded. Participants were recruited from three hospitals (oncology, respiratory medicine, and palliative care departments), two respiratory surgeries, and a palliative home care service in Munich, Germany.

\section{Data Collection}

Interviews were conducted by two trained and experienced interviewers (one physician not involved in patient care and one sociologist). The in-depth interviews were informal in style but followed the same topic guide. The main focuses of this guide were course of illness, experience living with breathlessness, impact on daily activities, family and social life, treatment experiences, and future expectations.

As the interviewed patients also participated in the longitudinal study described above, data on 
demographic and clinical characteristics (age, sex, marital status, main carer, place of care at study entry, specific diagnosis, and functional status) and breathlessness severity were collected as part of the baseline assessment for the longitudinal study.

All but one of the interviews were conducted in patients' homes. One patient was interviewed during an inpatient stay on a palliative care unit. The length of interviews varied from 20 minutes to 1 hour and 20 minutes. All interviews were audiotaped and transcribed verbatim.

\section{Data Analysis}

Framework analysis was employed to organize the data and identify emerging themes. It was chosen because it is a rule-governed, transparent, and structured approach and for its potential to undertake thematic and group analyses. Thus, quality criteria for qualitative research achieved throughout the research process served to ensure intersubjective traceability, reflexivity, and transparency of the research process (Mays \& Pope, 2000), as well as empirical anchoring.

A matrix-based approach framework analysis was developed by the National Centre for Social Research during the 1980s (Spencer et al., 2003) that involves developing a thematic framework through the independent repeated reading of interview transcripts and their systematic, peer group-reflected analysis. Richie and Spencer describe the following five steps of framework analysis: familiarization, identifying recurring and important themes, indexing, charting, analyzing, and interpreting (Spencer et al., 2003).
Primary analysis of data was conducted by the first author. Regular peer discussion (STS, CB) helped to adjust the categories identified in the primary data during the process of indexing and charting. The final framework reduced and structured the primary data. The framework was utilized to undertake thematic as well as (disease-related) group analyses. At this level of analysis, comparison between disease groups was realized.

\section{Ethical Approval}

Ethical approval was obtained from two research ethics committees-the College Research Ethics Committee at King's College London (CREC, number 05/06-69) and, as data collection took place in $\mathrm{Mu}-$ nich, the Research Ethics Committee of the University Hospital in Munich (number 079-06).

\section{RESULTS}

\section{Participants}

Ten COPD and eight lung cancer patients agreed to participate in the qualitative study. The two groups were similar with regard to demographic and clinical characteristics (see Table 2).

Two distinct types of crises were apparent from the data: breathlessness was experienced as a physical crisis-choking or an emergency situation-or as a psychological crisis-with panic, anxiety, and uncertainty.

Both cancer and COPD patients experienced breathlessness as a common symptom of their disease with specific triggers and impact on daily living.

Table 2. Demographic and clinical characteristics of 8 cancer and 10 COPD patients

\begin{tabular}{llcc}
\hline \hline Demographic characteristics & & Cancer $(n=8)$ & COPD (n=10) \\
\hline Age & Median (IQR) & $67(63.5-71)$ & $66.5(62-76)$ \\
Sex & Male/female & $5 / 3$ & $4 / 6$ \\
Marital status & Married & 6 & 4 \\
& With partner & 1 & 1 \\
& Divorced & 0 & 0 \\
& Widowed & 1 & 3 \\
Main carer & Single & 0 & 2 \\
& Spouse/partner & 6 & 3 \\
& Children/other relatives & 1 & 3 \\
Place of care at study entry & Friends & 0 & 1 \\
& Nobody & 1 & 3 \\
& Respiratory unit & 1 & 5 \\
Breathlessness severity Borg & Median (IQR) & - & 5 \\
Specific diagnosis & Number & 5 & - \\
Functional status (KPS) & Median (IQR) & 2 & - \\
& & $3.5(3-4.5)$ & COPD III/IV 5/5 \\
& Pncology unit & 6 (6-6)
\end{tabular}


However, the concomitant circumstances were different and affected experiences as well as patients' coping and adaptation mechanisms. Differences lay in the way patients perceived and responded to breath- lessness and the respective emerging crises. In the following, the experience of breathlessness and identified adaptation mechanisms are presented in more detail and illustrated in Figure 1.
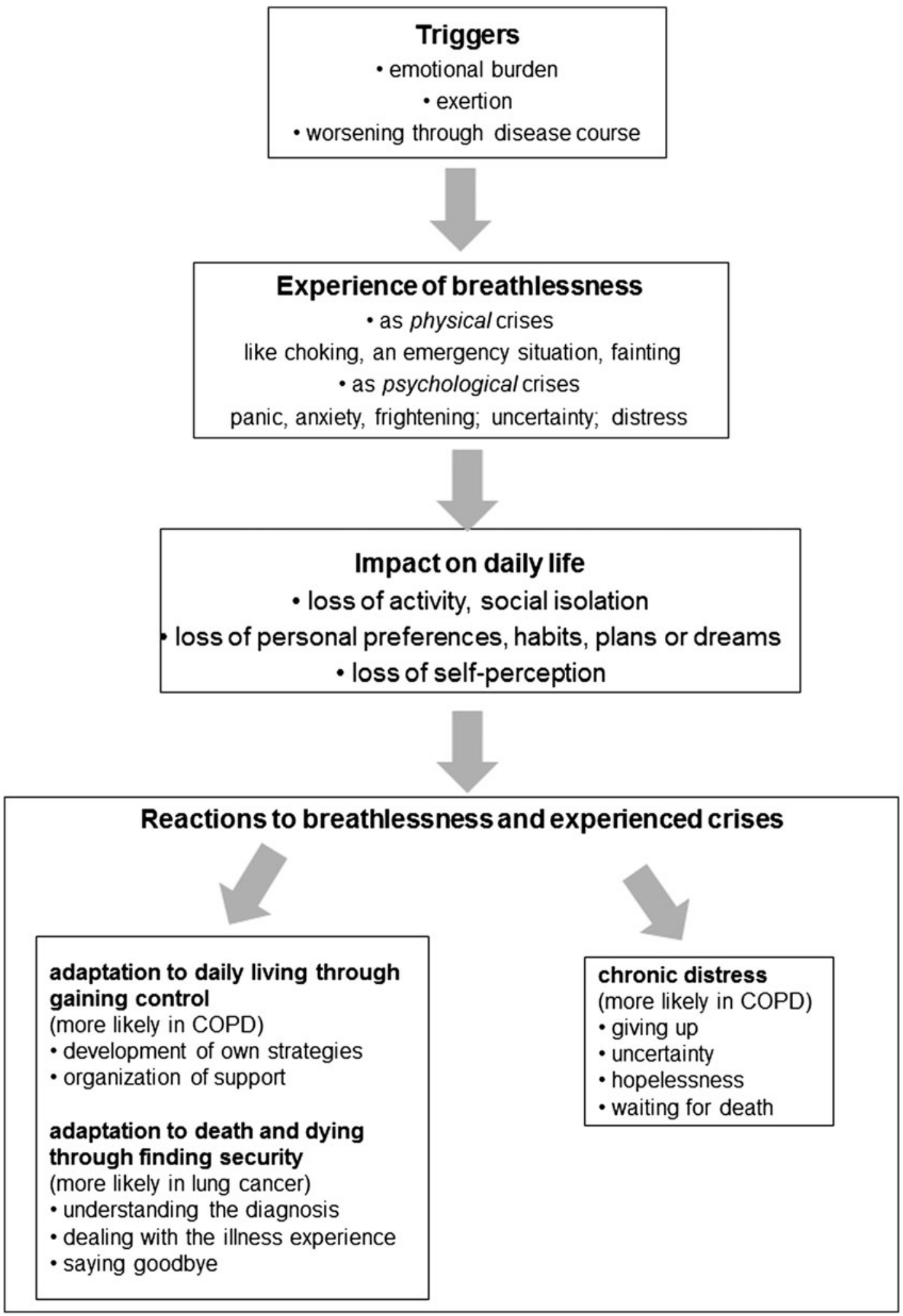

Fig. 1. Experience of breathlessness and patients' adaptations. 


\section{Experience of Breathlessness and Its Triggers}

The experience of breathlessness was predominantly described as a frightening and life-threatening situation with physical and psychological crises. Breathlessness as an emergency situation with a feeling of suffocation was known to both groups, with a tendency to be mentioned more often and explicitly by COPD patients. Descriptions of this emergency situation were dominated by images of agonizing, panic, or choking:

And you can't escape, and you get the feeling somebody holds you tight and strangles you. I think it is like somebody kills you and throttles you. [10901Ca]

It was a fight for life and death every nightmentally, not a real one, not a physical one. [2001COPD]

Breathlessness was thus an existential event causing anxiety and loss of control. Anxiety was experienced as an emotion causing breathlessness or exacerbating it. However, it was also a consequence of breathlessness, as one participant stated,

And then you get breathless, and it probably increases because you are becoming anxious, panicky. This is somehow like a chain reaction. It escalates. [3201COPD]

Various triggers led to breathlessness. Exertion was mentioned by all participants and described as worsening throughout the course of the disease. However, emotional burden and distressing situations were also specifically stressed by participants:

This strained me so much that I had half a nervous breakdown, and that is why I didn't get any air in. [6001Ca]

\section{Impact of Breathlessness on Daily Life}

All participants experienced a great impact of breathlessness on daily living, on their activities, and on their relationships. Halting physical activity and exertion was a common strategy to avoid triggers. In most cases, this led to loss of independence and social activities, also affecting the perception of the illness and their self-perception. Breathlessness became part of their daily living and was dictating their lives:

Yes, I live in a different way. In the past, I lived the way I wanted to, and today I live the way the illness allows it. I must adapt to the situation. [0301COPD]

Personal preferences or habits were lost and long planned or shared dreams destroyed:

The whole life has completely changed. (. . .) I love traveling, and I love life. In the past, I was out every day; this is not possible anymore. I can't do it anymore. [0301COPD]

It has changed a lot. All what we have planned. Now that I am retired, it came at the same time as being retired (...) Nothing! We couldn't realize anything! And I am not talking about the future anymore. And I don't have any plans anymore. [8301COPD]

\section{Reaction to Breathlessness and Crises: Adaptation to Daily Living}

COPD patients who had been living with their chronic condition for years referred to breathlessness as the most dominant symptom that had become an inherent part of their life. They either developed mechanisms of adaptation to these new circumstances or remained in a state of chronic distress.

Those who adapted to breathlessness and integrated it into their daily living often regained control over their lives either through dealing with the problems that occurred as a consequence of their breathlessness or through rearranging their everyday life in order to prevent these problems:

Well, this is my game where I say if I do more now and feed myself well and get exercise and what else you are meant to do that it lasts long, then I will have less of it when I am older, and most importantly the better I will die. [2001COPD]

They also developed own strategies and organized social and carers support:

And, you know, this is why I have this thing [a cushion] - to elevate myself so I have enough space for breathing. This is why I like cleaning the windows so much because I can stretch myself. [2901COPD]

Just friends, e.g., colleagues and friends, where I can easily say, if you go shopping, could you drop in? Or my colleague goes regularly to [town], which means I go shopping here, leave it in the office and he brings it here when he goes to [town]. [2001COPD]

Furthermore, participants who started to manage their illness in this way reported a special motivation 
to deal with breathlessness as well as emergent crises.

\section{Reaction to Breathlessness and Crises: Chronic Distress}

In some COPD patients, when patients had to change their lives, their social contacts, or their own behavior completely, they struggled to integrate these changes and consecutive losses into daily life. In consequence, they often did not manage to come out of the crisis they were experiencing. These participants described hopelessness and distressing situations and seemed to remain in some kind of chronic distress:

My brother sometimes takes me out for a ride, but otherwise life is not worth living. [2101COPD]

Others negotiated or repressed their illness condition.

It won't become worse; at least this is what I imagine. Otherwise, I can't do anything anymore if I can't breathe. [1901COPD]

None of the lung cancer patients spoke about breathlessness dominating their perspective of daily living.

\section{Reaction to Breathlessness and Crises: Adaptation to Death and Dying}

The dominant themes of participants suffering from advanced cancer were death and dying. They showed different ways of coping. Some accepted their approaching death and tried to say goodbye to their life and relatives, sometimes combined with the sorrow of leaving their family behind. They also expressed hopes and last wishes, for example, where they wanted to die or what they wanted to do for a last time:

I don't want to give my wife the feeling "he died there [in this bed]." She said to me, "If you die at home you die at home, but I accept your will that you want to die in hospital." [8901Ca]

This would be my wish to play golf again once more. [1001Ca]

Their main needs were to understand their diagnoses and to deal with their illness experiences:

We know that the cancer is definite. We know that we have to live with it. We are happy for every morning when we can say "good morning" and for every evening when we say "good night." We enjoy it. $[8901 \mathrm{Ca}]$
(. . .) and then anxiety, that I will suffocate later. (. . .) I am not afraid of dying or so. (. . .) Not that I will go voluntarily, but one has to be realistic. [10001Ca]

Further, most participating lung cancer patients suffered from their disease for a shorter time. They appeared to experience crises connected with uncertainty and searched for security to stabilize the situation:

Yes, this is difficult, because there is no real prognosis. Well, of course, you could say, in three, four, five weeks, this or that will happen, but this isn't the case. Yes, very uncertain, yes. [10401Ca]

If somebody says, "You can ring me anytime," this means for me security, as a patient. [8901Ca]

Presence of family members or informal carers was the most important nonprofessional support for patients with lung cancer:

Always in the house. Everything together. Everything I do, whether visiting the doctor or the therapist, I do together with her [wife]. [8301Ca]

Death and dying was only mentioned by two COPD patients in the interviews. One expressed hopelessness and distress describing the feeling of "waiting for death" [2101COPD]. The other was cared for in a palliative care unit and died 11 days later in a hospice:

One time, they say, she is dying, then they say, she will get better. Then they say again she will die and then better again. This game happened at least six times. This is very distressing for everybody. [00101 COPD]

\section{DISCUSSION}

Our study is one of the few comparative qualitative studies that explored and contrasted the experience of breathlessness of patients with COPD or lung cancer in the context of their illness. Both COPD and lung cancer patients experience physical and psychological crises associated with breathlessness. Crises at the end of life could be understood either as emergencies with a sudden start and a special pressure to act (Nauck \& Alt-Epping, 2008) or as distress, for example, a psychosocial emergency that could also become chronic (Broberger et al., 2007). Both types of crises were referred to by participants. Emergency situations were described more often and differentiated by COPD patients 
than by cancer patients. They seemed to have experienced more emergency situations and could describe them in more detail. They were also able to compare breathlessness attacks over the years and how they worsened. In a comparative study of patients suffering from cardiorespiratory diseases or cancer, Exley et al. (2005) suggested that COPD patients have little awareness of the seriousness of their condition, little knowledge about available services, and experience more difficulty in accessing them as well as difficulty in obtaining a clear prognosis, which leads to less organized treatment. This could cause more emergencies. Otherwise, the COPD patients in our study have had breathlessness for a longer time than the lung cancer patients and therefore had more experience, which they described in detail. They have developed various strategies to prevent exacerbations when coping with crises. Distress was, with regard to the losses and extent of limitations in social and daily living, much less preventable. The findings about breathlessness as leading to distress and the role of the illness context match the model of total dyspnea as suggested by several authors (e.g., Abernethy \& Wheeler, 2008; Gysels et al., 2007). Based on this model, breathlessness has to be understood as experience, influenced by the environment and, in turn, influencing the environment itself. This model was borrowed from the total pain model first described by Dame Cicely Saunders, and it adhered to the dimensions of physical symptoms, psychological concerns, social impact, and existential suffering (Saunders, 1964). All described dimensions were directly or indirectly expressed by the participants in our study. The results also show a connection between these dimensions, clearly pointed out in the influence of the context of the advanced illness and the different prospects.

Patients' reactions to the experienced crises differed, potentially due to contrasting (life) prospects. While COPD patients, who have the perspective of a longer life, under certain preconditions start to think of daily living with their condition and the symptom of breathlessness, lung cancer patients in a palliative situation more likely reach the point of $\mathrm{fa}$ cing death and dying. Gysels and Higginson (2011) found similar results in an English patient group where patients suffering from cancer, COPD, heart failure, or motor neuron disease (MND) shared the disabling effects of breathlessness but showed differences in the specific constraints of the illness and patients' experiences with the healthcare context and social environment. The prospects also seem to convey different strategies for COPD and cancer patients to cope with their crises. Lung cancer patients tried to find security within the uncertainty, while COPD patients tried to gain control over their breathlessness, their illness, and their daily living. This coping strategy, reflected in adaptation through time, is similar to the "acceptance of COPD" described by Pinnock and colleagues (2011). They stress that the more passive coping could be seen as adaptation to a way of life and not just as resignation. However, aside from this passive behavior of reacting, our results also show a more active way of becoming engaged in the situation.

Patients' reactions to crises caused by breathlessness and their adaptation processes could also derive from a combination of the illness experience, the duration of the disease, and the stage of disease. All 8 cancer patients died within months of the interview, whereas only 2 of the 10 COPD died within the same timeframe. Cancer patients often have less time to adapt to breathlessness, as it develops more quickly and is accompanied by an immediately life-threatening illness (Booth et al., 2003). Therefore, it is not surprising that cancer patients' reactions to breathlessness are clearly linked with death and dying. However, issues around death and dying were also raised as a topic by COPD patients, especially those who died shortly afterward.

These findings have implications for services dealing with breathless patients, be it in palliative care or respiratory or general medicine. First, breathlessness treatment must be given top priority not only to reduce physical symptom burden but also to lessen anxiety and psychological distress. Second, as breathlessness has psychosocial and existential dimensions, professionals should use the symptom experience to explore the deeper meaning and impact on patients' life perspectives, especially in the context of the type and stage of disease. Thus, the experience of breathlessness could be a starting point from which to gently explore fear of dying with cancer patients or address resources and problems in the daily activities of COPD patients. Psychosocial support should be offered to patients and address the areas of crisis, distress, and patients' coping mechanisms. Third, reactions to breathlessness might also be influenced by the patient's mood, and it is known that breathlessness is closely linked to anxiety and depression (Cleland et al., 2007). The patients in our study described a fear of suffocating and the anxiety following acute attacks. Both pharmacological and nonpharmacological interventions should be offered to patients to alleviate anxiety. Those who seem to live in chronic distress as a reaction to their disease and the experience of breathlessness should also be screened for depression in order not to overlook treatable mood disturbances. Fourth, carers play an important role in supporting patients. Although not a 
focus of these interviews, attending carers showed signs of distress themselves. The positive and negative effects of breathlessness on carers have been described before in greater detail (Gysels \& Higginson, 2009). Professionals need to be aware of carers' needs, should actively offer support, and not wait until they show signs of distress.

\section{STRENGTHS AND LIMITATIONS OF THE STUDY}

This qualitative study was embedded in a larger longitudinal cohort aiming to describe breathlessness trajectories. Although the cohort was much larger, we only had limited resources to conduct additional qualitative interviews. We nevertheless feel that a sample of 18 patients provides important information and that the inclusion of two different disease groups is a strength of our study. Also, we did not interview informal carers of breathless patients, but they occasionally attended the interviews and gave comments, which influenced the analyses. Listening to the experiences of informal carers is very important to meet their needs, but this was not within the scope of our study.

Patients participating in interviews were at different stages of their disease, which probably influenced their reactions to breathlessness and their coping styles. We had hoped to include patients in similar disease stages. It is known that breathlessness is a predictor for shorter survival in cancer patients (Geraci et al., 2006), but the prognosis is much more challenging for COPD patients (Rocker et al., 2007). Although COPD patients were at severe and very severe stages of their disease, their survival differed significantly from that of cancer patients. These data do not provide enough information to establish to what extent cancer patients adapted themselves and their daily living to their disease or the related illness experience earlier in the trajectory. However, the topics of death and dying are almost automatically linked to the diagnosis of lung cancer, but not necessarily to COPD.

\section{CONCLUSION}

The experience of breathlessness in advanced disease leads to physical and psychological crises. Understanding these crises and patients' reactions should be seen in the context of advanced illness. Even if patients with different conditions experience their symptoms (e.g., breathlessness) in a similar way, different (life) prospects will influence the meaning applied to the experience of breathlessness and the coping strategies developed over time. Therefore, professionals should react not only to patients' symptoms but also explore their deeper meaning and their impact on patients' life perspectives. Support needs to be tailored to patients' perspectives, and the experience of breathlessness could be a way to start talking about the fear of dying with lung cancer patients or addressing resources and problems in the daily activities of COPD patients. Further research should investigate the best ways to support these patients, providing interventions to help patients better address their needs and enhance coping strategies.

\section{ACKNOWLEDGMENTS}

This study was part of a Ph.D. fellowship of CB supported by Cicely Saunders International, UK, within the Breathlessness Research Program. We are grateful for the support of our colleagues who helped with recruitment of patients, namely, Drs. Weber and Duell from the Asklepios Fachkliniken Gauting and Drs. Bullemer and Schmidt and colleagues from the Interdisciplinary Centre of Palliative Medicine at Munich University Hospital and St. Christopher's Hospice Association, Munich. We are also indebted to Philip Anderson, Munich, who helped in conducting the interviews. IJH is a National Institutes for Health Research (UK) Senior Investigator.

\section{FUNDING}

This research was funded by Cicely Saunders International, London.

\section{CONTRIBUTIONS}

IJH obtained funding for the study; CB and IJH were responsible for the design of the study, ethics, and management; IJH, SB, and MG supervised the study; $\mathrm{CB}$ and PA collected data. CD analyzed the data under the supervision of CB and STS. CD drafted the paper with considerable input from $\mathrm{CB}$ and STS. All authors had full access to all of the data in the study and can take responsibility for the integrity of the data and the accuracy of the data analysis. $\mathrm{CB}$ is the guarantor.

\section{DECLARATION OF INTEREST}

The authors confirm that there is no conflict of interest.

\section{REFERENCES}

Abernethy, A.P. \& Wheeler, J.L. (2008). Total dyspnoea. Current Opinions in Supportive and Palliative Care, 2, 110-113.

American Thoracic Society (1999). Dyspnea: Mechanisms, assessment and management. A consensus statement. 
American Journal of Respiratory and Critical Care Medicine, 159, 321-340.

Bausewein, C., Booth, S., Gysels, M., et al. (2010a). Individual breathlessness trajectories do not match summary trajectories in advanced cancer and chronic obstructive pulmonary disease: Results from a longitudinal study. Palliative Medicine, 24(8), 777-786.

Bausewein, C., Booth, S., Gysels, M., et al. (2010b). Understanding breathlessness: Cross-sectional comparison of symptom burden and palliative care needs in chronic obstructive pulmonary disease and cancer. Journal of Palliative Medicine, 13, 1109-1118.

Booth, S., Silvester, S. \& Todd, C. (2003). Breathlessness in cancer and chronic obstructive pulmonary disease: Using a qualitative approach to describe the experience of patients and carers. Palliative \& Supportive Care, 1, $337-344$.

Booth, S., Bausewein, C., Higginson, I., et al. (2009). Pharmacological treatment of refractory breathlessness. Expert Review of Respiratory Medicine, 3, 21-36.

Broberger, E., Tishelman, C., von Essen, E.L., et al. (2007). Spontaneous reports of most distressing concerns in patients with inoperable lung cancer: At present, in retrospect and in comparison with EORTC-QLQ-C30 + LC13. Quality of Life Research, 16, 1635-1645.

Cleland, J.A., Lee, A.J. \& Hall, S. (2007). Associations of depression and anxiety with gender, age, health-related quality of life and symptoms in primary care COPD patients. Family Practice, 24, 217-223.

Exley, C., Field, D., Jones, L., et al. (2005). Palliative care in the community for cancer and end-stage cardiorespiratory disease: The views of patients, lay-carers and healthcare professionals. Palliative Medicine, 19, 76-83.

Geraci, J.M., Tsang, W., Valdres, R.V., et al. (2006). Progressive disease in patients with cancer presenting to an emergency room with acute symptoms predicts short-term mortality. Supportive Care in Cancer, 14, 1038-1045.

Gysels, M. \& Higginson, I.J. (2008). Access to services for patients with chronic obstructive pulmonary disease: The invisibility of breathlessness. Journal of Pain and Symptom Management, 36, 451-460.

Gysels, M.H. \& Higginson, I.J. (2009). Caring for a person in advanced illness and suffering from breathlessness at home: Threats and resources. Palliative \& Supportive Care, 7, 153-162.

Gysels, M. \& Higginson, I.J. (2010). The experience of breathlessness: The social course of chronic obstructive pulmonary disease. Journal of Pain and Symptom Management, 39, 555-563.
Gysels, M.H. \& Higginson, I.J. (2011). The lived experience of breathlessness and its implications for care: A qualitative comparison in cancer, COPD, heart failure and MND. BMC Palliative Care, 10, 15.

Gysels, M., Bausewein, C. \& Higginson, I.J. (2007). Experiences of breathlessness: A systematic review of the qualitative literature. Palliative \& Supportive Care, 5, 281-302.

Heinzer, M.M., Bish, C. \& Detwiler, R. (2003). Acute dyspnea as perceived by patients with chronic obstructive pulmonary disease. Clinical Nursing Research, 12, 85-101.

Lynn, J., Ely, E.W., Zhong, Z., et al. (2000). Living and dying with chronic obstructive pulmonary disease. Journal of the American Geriatrics Society, 48, S91-100.

Mays, N. \& Pope, C. (2000). Qualitative research in health care: Assessing quality in qualitative research. BMJ, $320,50-52$.

Nauck, F. \& Alt-Epping, B. (2008). Crises in palliative care: A comprehensive approach. The Lancet Oncology, 9, 1086-1091.

Pinnock, H., Kendall, M., Murray, S.A., et al. (2011). Living and dying with severe chronic obstructive pulmonary disease: Multi-perspective longitudinal qualitative study. BMJ, $342, \mathrm{~d} 142$.

Ramsenthaler, C., Scheve, C., Bausewein, C., et al. (2012). Typische Krisen und Bedürfnisse von Patienten mit COPD oder Lungenkrebs im letzten Lebensjahr-eine Literaturübersicht. Zeitschrift für Palliativmedizin, 13(3), 134-141.

Roberts, D.K., Thorne, S.E. \& Pearson, C. (1993). The experience of dyspnea in late-stage cancer: Patients' and nurses' perspectives. Cancer Nursing, 16(4), 310-320.

Rocker, G.M., Sinuff, T., Horton, R., et al. (2007). Advanced chronic obstructive pulmonary disease: Innovative approaches to palliation. Journal of Palliative Medicine, 10, 783-797.

Saunders, C. (1964). Care of patients suffering from terminal illness at St. Joseph's Hospice, Hackney, London. Nursing Mirror, 14, vii-x.

Solano, J.P., Gomes, B. \& Higginson, I.J. (2006). A comparison of symptom prevalence in far advanced cancer, AIDS, heart disease, chronic obstructive pulmonary disease and renal disease. Journal of Pain and Symptom Management, 31, 58-69.

Spencer, L., Ritchie, J. \& O'Connor, W. (2003). Carrying out qualitative analysis. In Qualitative research practice. J. Ritchie \& J. Lewis (eds.), pp. 219-262. London: Sage Publications. 